\title{
Evaluación de un carbón activado comercial en la remoción del colorante DB2
}

\section{Evaluation of a commercial activated carbon in the removal of the dye DB2}

\author{
Castellar O Grey Cecilia ${ }^{1 *}$ M.Sc, Viloria C Carlos Andres ${ }^{2}$ Ing Quim, Morrinson B Carlos Andres ${ }^{2}$ Ing \\ Quim, Angulo M Edgardo Ramon ${ }^{1}$ M.Sc, Zambrano AAlejandra María ${ }^{3}$ M.Sc. \\ 1Universidad del Atlántico, Ciencias Química, Facultad de Ciencias Básicas. Colombia. \\ ${ }^{2}$ Universidad del Atlántico, Colombia. \\ ${ }^{3}$ Ciencias Química, Universidad de la Costa, Barranquilla, Colombia.
}

\section{Keywords:}

Environment; organic compounds; adsorption; adsorption isotherms.

\section{Abstract}

Synthetic dyes used in the manufacture of textiles are an important class of organic compounds often found in bodies of water, as a result of its wide industrial use, becoming agents unfortunately affect the environment. The final disposition of these dyes to water resources should be avoided or minimized; for this purpose, various treatment techniques are used, the adsorption being one of the most used. In this study the direct navy blue dye stirred in aqueous solution onto granular activated carbon at $22^{\circ} \mathrm{C} \pm 1^{\circ} \mathrm{C}$. Batch experiments were conducted to determine the effect of the initial concentration $\left(7,0-65,0 \mathrm{mgL}^{-1}\right)$ and $\mathrm{pH}(5,0-9,0)$ on the adsorption capacity and removal percentage. The experimental data were fitted to models Freundlich adsorption isotherms and Langmuir. The maximum adsorption capacity achieved by the activated carbon was $2,22 \mathrm{mgg}^{-1}$ at $\mathrm{pH} 5,0$.

\section{Palabras Clave:}

Medio ambiente; compuestos orgánicos; adsorción; isotermas de adsorción.

INFORMACIÓN

Recibido: 08-03-2017;

Aceptado: 20-06-2017.

Correspondencia autor:

greycastellar@mail.uniatlantico.edu.co

\section{Resumen}

Los colorantes sintéticos usados en la fabricación de textiles son una clase importante de compuestos orgánicos que, con frecuencia, se encuentran en los cuerpos de agua, como resultado de su amplio uso industrial, convirtiéndose en agentes que desafortunadamente afectan el medio ambiente. La disposición final de estos colorantes a los recursos de agua se debe evitar o minimizar; para tal efecto, se utilizan varias técnicas de tratamiento, siendo la adsorción una de las más empleadas. En este estudio se removió el colorante azul marino directo en disolución acuosa sobre carbón activado granular comercial a $22 \pm 1^{\circ} \mathrm{C}$. Se realizaron experimentos por lote para determinar el efecto de la concentración inicial (7-65 $\left.\mathrm{mgL}^{-1}\right)$ y del $\mathrm{pH}(5,0-9,0)$ sobre la capacidad de adsorción y el porcentaje de remoción. Los datos experimentales se ajustaron a los modelos de isotermas de adsorción de Freundlich y Langmuir. La capacidad de adsorción máxima lograda por el carbón activado fue de 2,22 $\mathrm{mgg}^{-1}$ a pH 5,0. 


\section{Introducción}

Las condiciones del medio ambiente no son las mejores. Día a día, los cuerpos de aguas son contaminados por diversas sustancias químicas provenientes principalmente del sector industrial. En este sentido, la industria textil tiene un aporte relevante, pues generan grandes volúmenes de aguas residuales contaminadas con colorantes, que debido a su alto peso molecular, sus estructuras complejas y especialmente a su alta solubilidad en agua muestran gran persistencia en el ambiente (BALDERAS et al., 2006), generando serios problemas por las implicaciones que tiene tanto para la salud pública como para los ecosistemas. En efecto, muchos colorantes azo y sus productos de degradación (aminas aromáticas) pueden generar alergias, y en el peor de los casos mutaciones o cáncer. Por otro lado, la descarga de estas sustancias a los sistemas acuáticos impide el desarrollo normal de las plantas debido a que inhiben la fotosíntesis (CERVANTES, 2008).

Las técnicas más empleadas en la eliminación de colorantes en aguas residuales consisten en métodos químicos, biológicos y físicos. El tratamiento químico utiliza agentes coagulantes o floculantes para eliminar el color, pero no es muy eficaz para colorantes que son muy solubles (SHI et al., 2007; ANGULO et al., 2013). Por otro lado, el tratamiento biológico tiene dificultades para eliminar colorantes de efluentes de manera continua, además se requiere de tiempos más prolongados en los procesos de decoloración (ROBINSON et al., 2001) y, por último, los tratamientos físicos, como la filtración por membrana, la destrucción electroquímica, la irradiación, la ozonización y la adsorción, entre otros, se usan ampliamente para el tratamiento de colorantes en aguas residuales. La mayoría de estos procesos son costosos y conducen a la generación de lodos o a la formación de subproductos (HAMEED y EL-KHAIARY, 2008); sin embargo, se conoce que la adsorción es uno de los métodos más eficaces, sobre todo si el adsorbente es económico y muestra una alta capacidad de adsorción.

Esta investigación evaluó la técnica de la adsorción para la remoción del colorante azul marino directo, empleando carbón activado granular comercial como material adsorbente en un proceso por lote. El efecto de la concentración inicial y el $\mathrm{pH}$, a temperatura y agitación constante fueron objeto de análisis. Los resultados experimentales se ajustaron a dos modelos de isotermas de adsorción convencionales.

\section{Materiales y Métodos}

Caracterización del carbón activado: las características de textura como el área superficial y el volumen de poro se determinó a partir de isotermas de adsorción-desorción de nitrógeno a $77 \mathrm{~K}$, aplicando el método de Brunauer, Emmett y Teller (BET). Se utilizó un analizador de área superficial y porosidad Micromeritics Gemini VII 2390 a. Se realizaron análisis próximo y elemental siguiendo las normas ASTM. La cantidad total de grupos funcionales ácidos orgánicos representados en ácidos carboxílicos, fenoles y lactonas presentes en el carbón activado se determinó por titulación con una serie de bases de diferente fortaleza siguiendo un procedimiento modificado basado en el método de Böehm (DE CELIS et al., 2009; NUNELL et al., 2012).

Estudio por lotes: el carbón activado se lavó con agua destilada y, posteriormente, se secó a $100^{\circ} \mathrm{C}$, durante 12 horas. Se preparó una disolución "stock" de $764 \mathrm{mgL}^{-1}$ del colorante azul marino directo (de aquí en adelante se denominará DB2 por su identificación según The Color Index), disolviendo la cantidad requerida en agua destilada. A partir de la disolución "stock", se prepararon diluciones de concentraciones entre los $7 \mathrm{mgL}^{-1}$ hasta los $65 \mathrm{mgL}^{-1}$, ajustándole el $\mathrm{pH}$ a valores comprendidos entre 5,0-9,0 $\pm 0,1$ con $\mathrm{HCl}$ y $\mathrm{NaOH} 0,1 \mathrm{M}$. La temperatura se mantuvo a condiciones de laboratorio $\left(22 \pm 1^{\circ} \mathrm{C}\right)$. A $1000 \mathrm{mg}$ del carbón activado se le adicionó $100 \mathrm{~mL}$ de cada una de las diluciones. Luego las muestras se colocaron en un agitador horizontal a 120 rpm por 12 horas, tiempo estimado para alcanzar la condición de equilibrio (AHMAD et al., 2007). Finalizada la agitación, se separó el carbón activado por filtración y se analizó la concentración final del colorante, usando la curva de calibración obtenida a través del espectrofotómetro marca THERMO SCIENTIFIC modelo EVOLUTION 605 .

Evaluación de la capacidad de adsorción: a partir de los datos obtenidos de los experimentos de equilibrio, se calculó el porcentaje de remoción de DB2 para cada uno de los sistemas estudiados mediante la Ecuación 1.

$\% E_{R}=\left(\frac{C_{o}-C_{e}}{C_{o}}\right) 100$

Donde $\mathrm{C}_{0}\left(\mathrm{mgL}^{-1}\right)$ es la concentración inicial de DB2 y $\mathrm{C}_{\mathrm{e}}\left(\mathrm{mgL}^{-1}\right)$ es la concentración en equilibrio. La capacidad de adsorción del carbón activado en el equilibrio $\mathrm{q}_{\mathrm{e}}\left(\mathrm{mgg}^{-1}\right)$ se calculó usando la Ecuación 2.

$q_{e}=\frac{\left(C_{o}-C_{e}\right) V}{W}$

Donde $\mathrm{V}(\mathrm{L})$ es el volumen de la disolución y $\mathrm{W}(\mathrm{g})$ es la masa del adsorbente. 


\section{Resultados y Discusión}

Caracterización del carbón activado: las propiedades fisicoquímicas tales como área superficial específica, volumen total de poro, material volátil, cenizas, contenido de carbono y grupos superficiales ácidos se muestran en la Tabla 1 (CASTELLAR et al., 2013).

Tabla 1. Principales características del carbón activado.

\begin{tabular}{lc}
\hline \multicolumn{1}{c}{ Parámetro } & Valor \\
\hline Área superficial $\left(\mathrm{m}^{2} \mathrm{~g}^{-1}\right)$ (basado en BET) & 587 \\
Volumen total de poro $\left(\mathrm{cm}^{3} \mathrm{~g}^{-1}\right)$ & 0,67 \\
Contenido de cenizas (base seca) (\%) & 14,5 \\
Contenido de humedad (\%) & 5,9 \\
Material volátil (\%) & 8,2 \\
& Análisis elemental (\%) \\
$\mathrm{C}$ & \\
$\mathrm{H}$ & 77,2 \\
$\mathrm{~N}$ & 2,8 \\
O (por diferencia) & 0,9 \\
$\mathrm{~S}$ & 3,9 \\
& Grupos funcionales ácidos (meqg-1) \\
Carboxílicos & 0,7 \\
Fenólicos & 0,50 \\
Lactónicos & 0,80 \\
\hline Total grupos ácidos & 0,20 \\
\hline
\end{tabular}

El área superficial del carbón activado comercial empleado en este estudio puede considerarse baja, considerando que ésta es una de las características más importante en este tipo de materiales. La capacidad de adsorción de los materiales no solamente se puede interpretar en términos de sus propiedades físicas, sino que también influye su química de superficie (BARATA et al., 2006), característica que contribuye con la eficiencia o rendimiento como adsorbente (BANSAL et al., 1988). La química de superficie depende de la presencia de heteroátomos, principalmente oxígeno (Tabla 1), que forma grupos funcionales orgánicos, como ácidos carboxílicos, lactonas, fenoles, carbonilos, quinonas, aldehídos, éteres, anhídridos entre otros (BOEHM, 1994), que pueden ser tanto ácidos como básicos $\mathrm{y}$, dependiendo del $\mathrm{pH}$ de la solución, afectan la capacidad de adsorción. El alto contenido de cenizas, humedad y material volátil presentes en el carbón activado empleado, disminuyen la capacidad efectiva como adsorbente.

Evaluación de la capacidad de adsorción: en la Fig. 1a se observa que el porcentaje de adsorción disminuye cuando se incrementa la concentración inicial, mientras que, la capacidad de adsorción (Fig. 1b) aumenta para todos los $\mathrm{pH}$ estudiados. En un estudio por lotes se mantiene el mismo volumen de disolución, por tal motivo, a bajas concentraciones la cantidad de colorante es menor que a altas concentraciones; manteniendo la misma masa de adsorbente, se removerá gran parte del colorante o su totalidad, quedando aún sitios disponibles para la adsorción, que serán ocupados en la medida que la concentración aumenta.

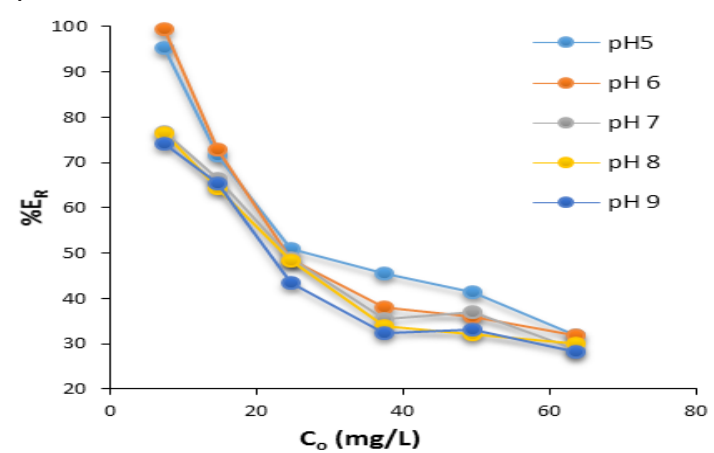

(a)
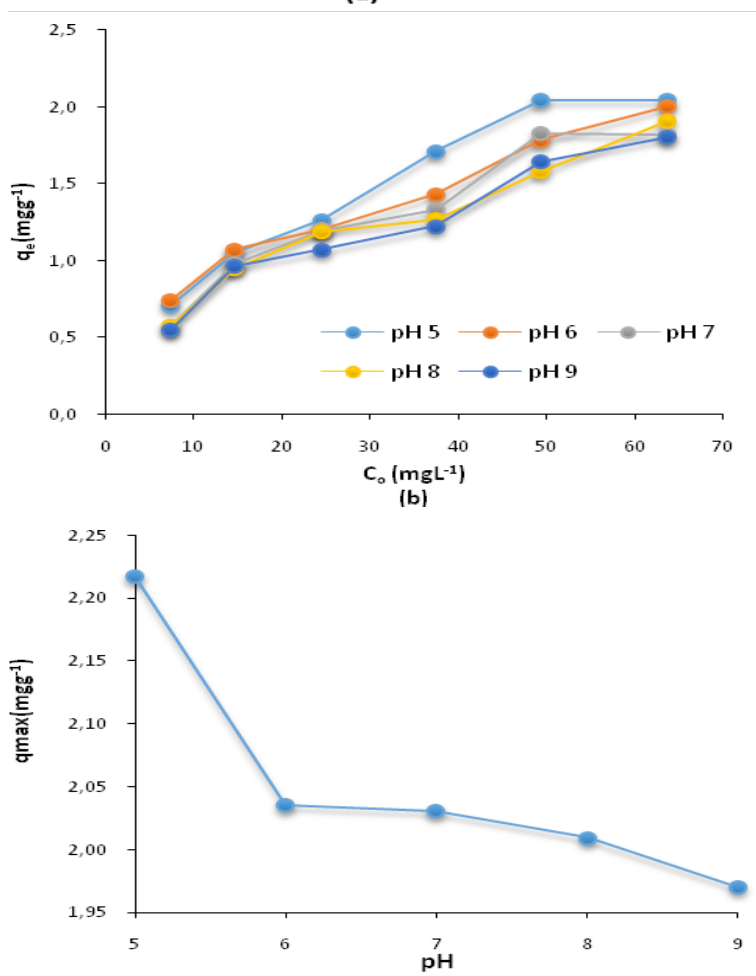

(c)

Figura 1. (a) Efecto de la concentración inicial de DB2 sobre el porcentaje de remoción, (b) efecto de la concentración inicial sobre la capacidad de adsorción y (c) efecto del pH sobre la capacidad máxima de adsorción.

Efecto del pH: el pH de la disolución es un parámetro importante en el proceso de adsorción ya que puede afectar la interacción de los grupos funcionales superficiales del adsorbato y el adsorbente. Además, las propiedades del carbón activado y de los colorantes podrán indicar si la adsorción es favorable en disoluciones ácidas o básicas (ALMEIDA et al., 2012). 
Para el estudio del efecto del pH sobre la capacidad de adsorción del colorante DB2 se realizaron experimentos a diferentes $\mathrm{pH}(5,6,7,8$ y 9$)$ de las disoluciones iniciales. De la Fig. 1c se observa que cuando se aumenta el $\mathrm{pH}$, la capacidad máxima de adsorción disminuye. De los resultados obtenidos y realizando los cálculos correspondientes se encontró que a $\mathrm{pH}$ 5 la capacidad máxima de adsorción fue 2,22 $\mathrm{mgg}^{-1}$, mientras que, a pH 9 fue $1,97 \mathrm{mgg}^{-1}$ (Tabla 2). Este comportamiento permite sugerir que en disolución acuosa los grupos sulfonatos $\left(-\mathrm{SO}_{3} \mathrm{Na}\right)$ del colorante se disocian y se convierten en aniones. A pH ácido es posible que se formen centros electrofílicos que pueden generar atracciones de naturaleza electrostática favoreciendo su adsorción (Figura 2). Con el aumento del $\mathrm{pH}$ de la disolución, los sitios activos con carga negativa aumentan, situación que no favorece la adsorción del colorante debido a la repulsión electrostática (DOTTO et al., 2009; NAKBANPOTE et al., 2005; DAWOOD y SEN, 2014).
Isotermas de adsorción: con el propósito de describir el equilibrio del colorante entre las fases sólida y liquida, se ajustó los datos experimentales a dos modelos de isotermas de adsorción: Langmuir y Freundlich. El modelo de Langmuir se expresa mediante la Ecuación 3.

$q_{e}=\left(\frac{q_{\max } b C_{e}}{1+b C_{e}}\right)$

Donde $\mathrm{q}_{\max }$ y $\mathrm{b}$ son las constantes de Langmuir, y representan la capacidad máxima de adsorción $\left(\mathrm{mgg}^{-1}\right)$ del adsorbente y la constante de energía relacionada con el calor de adsorción respectivamente $\left(\mathrm{dm}^{3} \mathrm{mg}^{-1}\right)$ (AKSU y GÖNEN, 2004). La figura 3 muestra los ajustes de los datos experimentales a la isoterma de Langmuir para cada $\mathrm{pH}$. Los valores de $\mathrm{q}_{\max }$ y $\mathrm{b}$ se determinan con la pendiente y la ordenada al origen de cada una de las líneas rectas.

Figura 2. (a) Grupo carboxílico presente en la superficie del carbón, (b) disociación de los grupos sulfonatos del colorante y (c) mecanismo sugerido de atracción electroestática con el grupo carboxílico. Tomado y adaptado de CHEUNG et al. (2004).<smiles>CC(C)C(C(=O)O)C(C)C</smiles>

(a)<smiles>Nc1ccc2cc([N+](=O)[O-])c(N=Nc3ccc(-c4ccc(N=Nc5c(O)cc6cc(O[N+](=O)[O-])cc(N)c6c5O)cc4)cc3)c(O)c2c1</smiles>

(b)

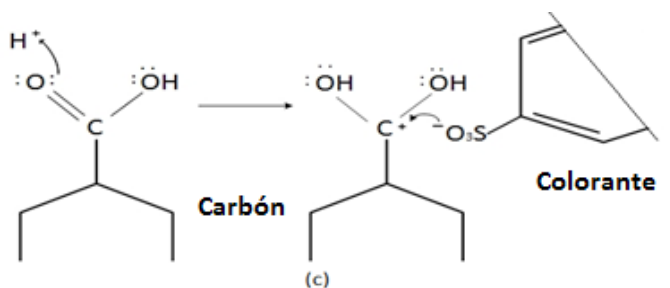

Tabla 2. Constantes de Freundlich y Langmuir.

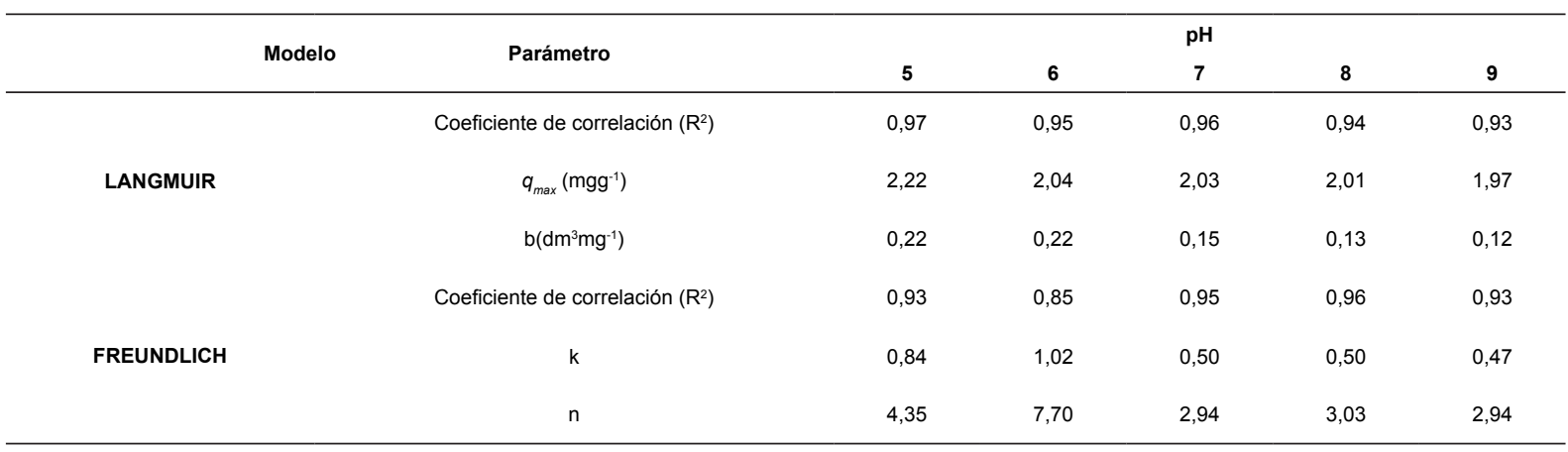


Figura 3. Datos experimentales ajustados a la isoterma de Langmuir.
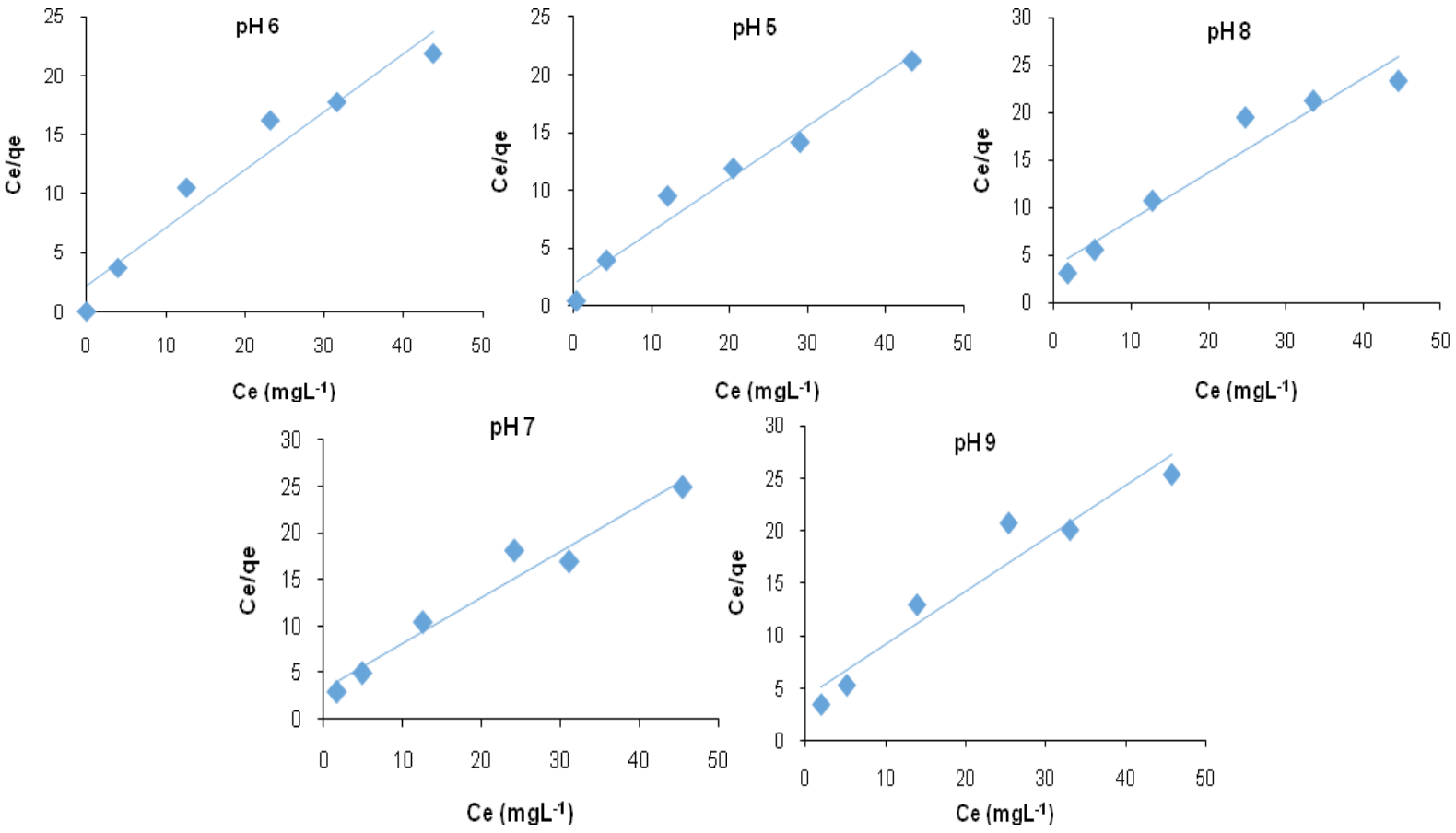

Freundlich encontró una relación de tipo exponencial entre la cantidad de soluto adsorbido y la concentración en equilibrio, el cual se expresa mediante la Ecuación 4.

$$
q_{e}=k C_{e}^{\frac{1}{n}}
$$

Donde $\mathrm{q}_{\mathrm{e}}\left(\mathrm{mgg}^{-1}\right)$ es la cantidad de adsorbible removido por unidad de masa de adsorbente, $\mathrm{C}_{\mathrm{e}}$ es la concentración en el equilibrio $\left(\mathrm{mgdm}^{-3}\right), \mathrm{K}$ es la constante de equilibrio $\left(\mathrm{mgg}^{-1}\left(\mathrm{dm}^{3} \mathrm{mg}^{-1}\right)^{1 / 1 n}\right)$ y $\mathrm{n}$ es una constante relacionada con la afinidad entre el adsorbente y el soluto. La Fig. 4 muestra el ajuste de los datos experimentales a la isoterma de Freundlich y la Tabla 2 resume los valores de los parámetros de ambos modelos.

Aunque los valores del coeficiente de correlación lineal $\mathrm{R}^{2}$ (Tabla 2) muestran un mejor ajuste al modelo de isoterma de Langmuir, son muy cercanos al modelo de Freundlich, lo cual sugiere que hay una adsorción en monocapa y multicapa sobre una superficie heterogénea. Los valores de $n$ que se encontraron en este estudio oscilaron entre 2,94 y 7,70 , esto indica, que el colorante DB2 se adsorbe favorablemente sobre el carbón activado comercial.
A través del modelo de Langmuir se determinó la capacidad máxima de adsorción $\left(\mathrm{q}_{\max }\right)$ del colorante sobre el carbón activado. Los resultados que se obtuvieron (1,97-2,22 $\left.\mathrm{mgg}^{-1}\right)$ muestran que es menor, comparado con otros materiales adsorbentes. Es posible que la estructura compleja y alta masa molar molecular del colorante DB2 dificultan su transporte y difusión en los poros del carbón activado afectando la capacidad de adsorción (DERRICHE et al., 2013).

\section{Conclusiones}

La metodología experimental desarrollada en esta investigación consistió en un estudio por lotes para determinar la capacidad de adsorción de un carbón activado comercial en la remoción del colorante DB2 en disolución acuosa bajo condiciones de temperatura, agitación y masa de adsorbente constantes, a diferentes $\mathrm{pH}$ y concentración inicial. Los resultados obtenidos permiten formular las siguientes conclusiones: el carbón activado comercial que se empleó tiene una química superficial heterogénea, con un área superficial y volumen de poro aceptable en comparación con otros materiales adsorbentes. Los datos experimentales se ajustan a los modelos de isotermas de adsorción de Langmuir y Freundlich. La capacidad máxima de adsorción obtenida fue de 2,22 $\mathrm{mgg}^{-1}$ a pH 5 . 
Figura 4. Datos experimentales ajustados a la isoterma de Freundlich.

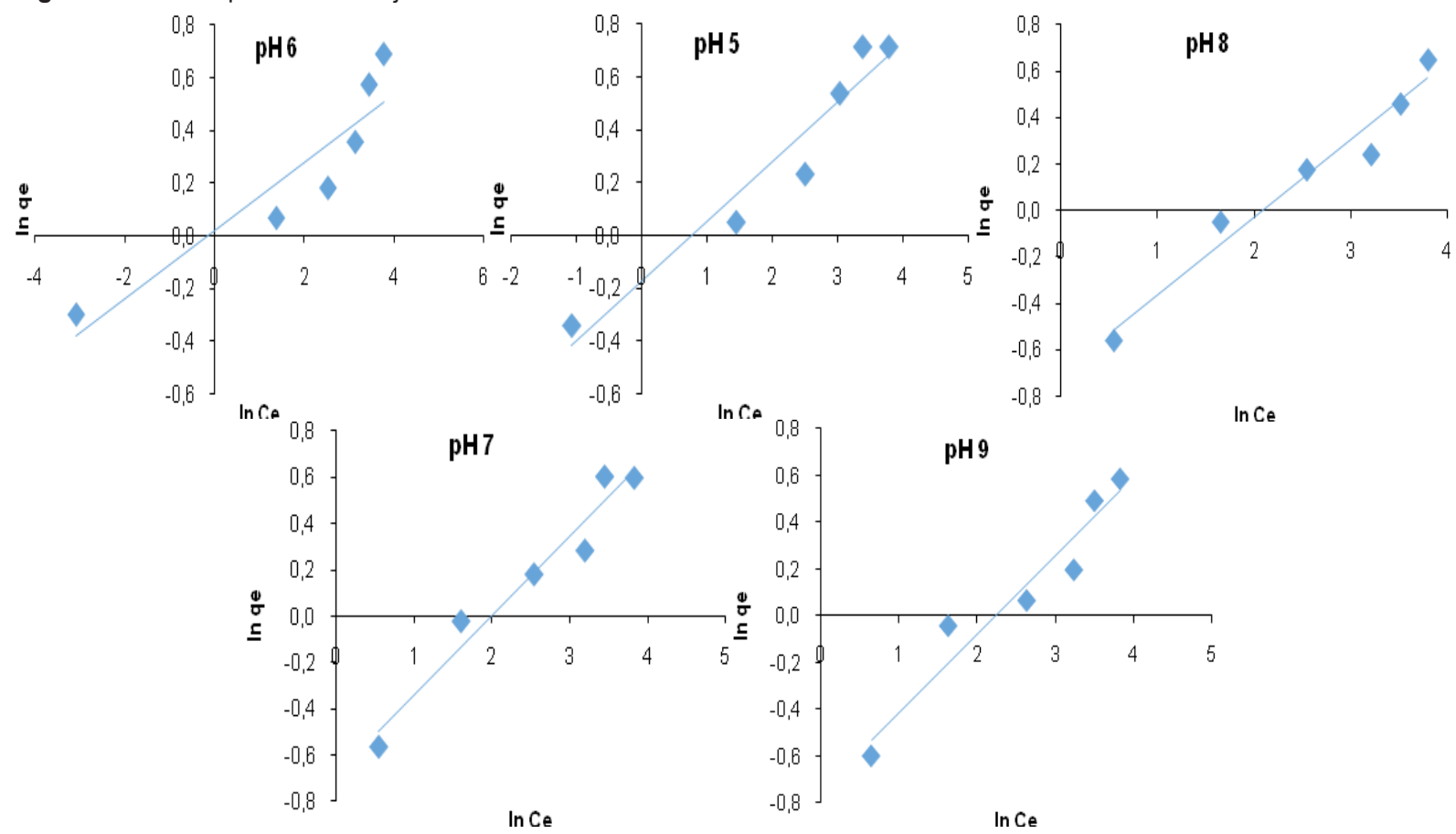

\section{Referencias}

AHMAD, A.L.; LOH, M.M.; AZIZ, J.A. 2007. Preparation and characterizacion of activated carbon from oil palm wood and its evaluation on methylene blue adsorption. Dyes and Pigments 75(2):263-272.

AKSU, Z.; GÖNEN, F. 2004. Biosorption of phenol by immobilized activated sludge in a continuous packed bed: prediction of breakthrough curves. Process Biochemistry 39(5):599-613.

ALMEIDA, V. C.; CAZETTA, A. L.; GARCIA, E. E.; GAUZE, G. F.; COSTA, W. F.; MARTINS, A. C.; VARGAS, A. M. 2012. Kinetic and equilibrium studies: Adsorption of food dyes Acid Yellow 6, Acid Yellow 23, and Acid Red 18 on activated carbon from flamboyant pods. Chemical Engineering Journal 65(4):243-250.

ANGULO, M. E.; CASTELLAR, O. G.; CHARRIS, F.D.; ZAMBRANO, A.A. 2013. Equilibrio de adsorción del colorante azul de metileno sobre carbón activado. Revista U.D.C.AActualidad \& Divulgación Científica 16(1):263-271.

BALDERAS, P.; BARRERA, C.; LUJANO, A.; RAMÍREZ, M. T.; ROA, G. 2006. Remoción de colorantes de aguas provenientes de industrias textiles usando electrocoagulación. Universidad Autónoma del Estado de México, Facultad de Química. MÉXICO.

BANSAL, R.C.; DONNET, J.B.; STOECKLI, F.1988. Activated Carbon. Ed. Marcel Dekker. Nueva York.

BARATA, S.A.; FARIA, P.C.; FONSECA, I.M.; PEREIRA, J.C.V.; PEREIRA, F.R.; ÓRFAO, J.M.; SILVA, A.I.M. 2006. Adsorption of a reactive dye on chemically modified activated carbons-influence of $\mathrm{pH}$. Colloid Interface Science 296(2):480-489. 
BOEHM, H.P. 1994. Some aspects of the surface chemistry of carbon blacks and other carbons. Carbon 32(5):759769.

CASTELLAR, O.G.; CARDOZO, A.B.; SUAREZ, G.J.; VEGA, T.J. 2013. Adsorción por lote y en una columna de lecho fijo del colorante B39 sobre carbón activado granular. Prospectiva 11(1):66-75.

CERVANTES,C.F.J. 2008. Reducción de colorantes azo por distintos grupos microbianos en consorcios anaerobios. BioTecnología 12(3):6-20.

CHEUNG, W.H.;McKAY, G.;SZETO, Y.S.; WONG, Y.C. 2004. Adsorption of acid dyes on chitosan-equilibrium isotherm analyses. Process Biochemistry 39(6):693-702.

DAWOOD, S.; SEN, T.K. 2012. Removal of anionic dye Congo red from aqueous solution by raw pine and acidtreated pine cone powder as adsorbent: Equilibrium, thermodynamic, kinetics, mechanism and process design. Water Research 46(6):1933-1946.

DE CELIS, J.; AMADEO, N. E.; CUKIERMAN, A. L. 2009. In situ modification of activated carbons developed from a native invasive wood on removal of trace toxic metals from wastewater. Journal of Hazardous Materials 161(1):217-223.

DERRICHE, Z.; DJAMEL, B.K.; KACHA, S.; KAMECHE, M. 2013. Adsorption kinetics of some textile dyes onto granular activated carbon. Journal of Environmental Chemical Engineering 1(3):496-503.

DOTTO, G.L.; GONÇALVES, J.O.; PICCIN, J.S.; PINTO, L.A.A.; VIEIRA, M.L.G. 2009. Adsorption of FD\&C Red No. 40 by chitosan: Isotherms analysis. Journal of Food Engineering 95 (1):16-20.

HAMEED, H.B.; EL-KHAIARY, M.I. 2008. Equilibrium, kinetics and mechanism of malachite green adsorption activated carbon prepared from bamboo by $\mathrm{K}_{2} \mathrm{CO}_{3}$ activation and subsequent gasification with $\mathrm{CO}_{2}$. Journal of Hazardous Materials 157(2-3):344-351.

NUNELL, G.V.; FERNÁNDEZ, M.E.; BONELLI, P.R.; CUKIERMAN, A.L. 2012. Conversion of biomass from an invasive species into activated carbons for removal of nitrate from wastewater. Biomass and Bioenergy 44:87-95.

ROBINSON, T.; McMULLAN, G.; MARCHANT, R.; NIGAM, P. 2001. Remediation of dyes in textile effluent: a critical review on current treatment technologies. Bioresource Technology 77(3):247-255.

SAKKAYAWONG, N.; THIRAVETYAN, P.; NAKBANPOTE, W. 2005. Adsorption mechanism of synthetic reactive dye wastewater by chitosan. Journal of Colloid and Interface Science, 286(1): 36-42.

SHI, B.Y.; LI, G.H; WANGS, D.S.; FENG, C.H.; TANG, H.X. 2007. Removal of direct dyes by coagulation: the performance of performance polymeric aluminum species. Journal of Hazardous Materials 143:567-574. 